\title{
The number of lymph nodes identified in a simple pancreatoduodenectomy specimen: comparison of conventional vs orange-peeling approach in pathologic assessment
}

\author{
N Volkan Adsay ${ }^{1}$, Olca Basturk ${ }^{2}$, Deniz Altinel ${ }^{1}$, Fayyaz Khanani ${ }^{3}$, Ipek Coban ${ }^{1}$, \\ Donald W Weaver ${ }^{4}$, David A Kooby ${ }^{5}$, Juan M Sarmiento ${ }^{5}$ and Charles Staley ${ }^{5}$ \\ ${ }^{1}$ Department of Pathology, Winship Cancer Institute, Emory University School of Medicine, Atlanta, GA, \\ USA; ${ }^{2}$ Department of Pathology, New York University, New York, NY, USA; ${ }^{3}$ Department of Pathology, \\ Karmanos Cancer Institute, Wayne State University School of Medicine, Detroit, MI, USA; ${ }^{4}$ Department of \\ Surgery, Karmanos Cancer Institute, Wayne State University School of Medicine, Detroit, MI, USA and \\ ${ }^{5}$ Department of Surgery, Winship Cancer Institute, Emory University School of Medicine, Atlanta, GA, USA
}

\begin{abstract}
Lymph node status is one of the most important predictors of survival in resectable pancreatic ductal adenocarcinoma; therefore, thorough lymph node evaluation is a critical assessment in pancreatoduodenectomy specimens. There is considerable variability in pancreatoduodenectomy specimens processed histologically. This study compares two approaches of lymph node dissection and evaluation (standard vs orange peeling) of pancreatoduodenectomy specimens. A different approach to dissection of pancreatoduodenectomy specimens was designed to optimize lymph node harvesting: All peripancreatic soft tissues were removed in an orange-peeling manner before further dissection of the pancreatic head. This approach was applied to 52 consecutive pancreatoduodenectomy specimens performed for ductal adenocarcinoma at two institutions. Specimen dissection was otherwise performed routinely. Overall number of lymph nodes harvested, number of positive lymph nodes, and their anatomic distribution were analyzed and compared with cases that had been dissected by the conventional approach. The mean number of lymph nodes identified by the orange-peeling approach was 14.1 (by institution, 13.8 and 14.4), as opposed to 6.1 (by institution, 7 and 5.3 ) in cases processed by conventional approach $(P=\mathbf{0 . 0 0 0 1})$. The number of lymph node-positive cases also increased substantially from $50 \%$ (by institution, 54 and $46 \%$ ) in the conventional method to $73 \%$ (by institution, 88 and $58 \%$ ) in the orange-peeling method $(P=0.02)$. The orange-peeling method of lymph node harvest in pancreatoduodenectomy specimens for ductal adenocarcinoma enhances overall and positive lymph node yield and optimizes ductal adenocarcinoma staging. Therefore, lymph node harvest by the orange-peeling method should be performed routinely before specimen sectioning in assessment of pancreatoduodenectomy for ductal adenocarcinoma.
\end{abstract}

Modern Pathology (2009) 22, 107-112; doi:10.1038/modpathol.2008.167; published online 26 September 2008

Keywords: lymph node; pancreas; pancreatoduodenectomy; grossing

Although pancreatic ductal adenocarcinoma is a uniformly fatal disease, ${ }^{1}$ there are, nevertheless, substantial differences in survival rates of pancreatic cancer by stage. ${ }^{2-4}$ It has been widely documented in the literature that lymph node status is one of

Correspondence: Dr NV Adsay, MD, Department of Pathology, Winship Cancer Institute, Emory University Hospital, Room H185-B, 1364 Clifton Road NE, Atlanta, GA 30322, USA.

E-mail: volkan.adsay@emory.edu

This study was presented in part at the annual meeting of the United States and Canadian Academy of Pathology in Vancouver, March 2004.

Received 31 March 2008; revised and accepted 02 June 2008; published online 26 September 2008 the most important independent prognostic factors of survival, in particular, for resectable cases. ${ }^{2-11}$ More importantly, recent studies have shown that the predictive value of lymph node status is directly proportional to the number of identified lymph nodes; staging might be inadequate if less than 15 lymph nodes are examined. ${ }^{12}$ Commission of Cancer of the American College of Surgeons is currently establishing guidelines for standards of practice for management of ductal adenocarcinoma. One of the issues under discussion is the number of lymph nodes in a pancreatoduodenectomy specimen (Dr Karl Y. Bilimoria and Dr Keith Lillemoe, personal communication, December 2007). 
At the same time, pancreatoduodenectomy specimens are complex to evaluate, and identification of margins and lymph nodes can be problematic. Most pathology laboratories have limited experience with these specimens, as these operations are performed infrequently at many institutions. The current approach to dissection of these specimens is to section the pancreatic head first and then search for the lymph nodes.

Currently there is no consensus on the optimal number of lymph nodes to examine to accurately stage node-negative ductal adenocarcinoma after pancreatoduodenectomy. Presumably many patients are understaged, as is evidenced by the variability of histologic lymph node counts in the literature. ${ }^{8,13,14}$ Data from two large Surveillance, Epidemiology, and End Results (SEER) database reviews of pancreatic cancer show that the median number of assessed lymph nodes was $7 .^{8,12}$

This study was performed to determine the number of lymph nodes typically present in pancreatoduodenectomy specimens, and to assess a potentially better approach to dissection of pancreatoduodenectomies to optimize lymph node yield and staging, while maintaining feasibility in daily practice.

\section{Materials and methods}

A modified approach to dissection of pancreatoduodenectomy specimens (described below) was applied to 52 pancreatoduodenectomy specimens performed at either (1) Wayne State University and Karmanos Cancer Institute (26 cases; 2001-2003) or (2) Emory University and Winship Cancer Institute (26 cases, 2007). Data from two separate institutions were included to minimize institutional and population-based biases. All cases were performed for a diagnosis of conventional ductal adenocarcinoma of the pancreas. Other periampullary cancers (ampullary, biliary, and duodenal carcinoma) were not included.

The number of lymph nodes identifiable in a routine pancreatoduodenectomy specimen was investigated by careful dissection of lymph nodes in the gross room. An orange-peeling approach was designed to ensure complete evaluation of potential lymph node sites. Accordingly, the peripancreatic soft tissues were dissected after the main three margins (common bile duct margin, retroperitoneal margin, and pancreatic neck margin) were obtained, but before sectioning of the pancreatic head (Figure 1). Peripancreatic soft tissues were subsequently shaved off and separated into eight
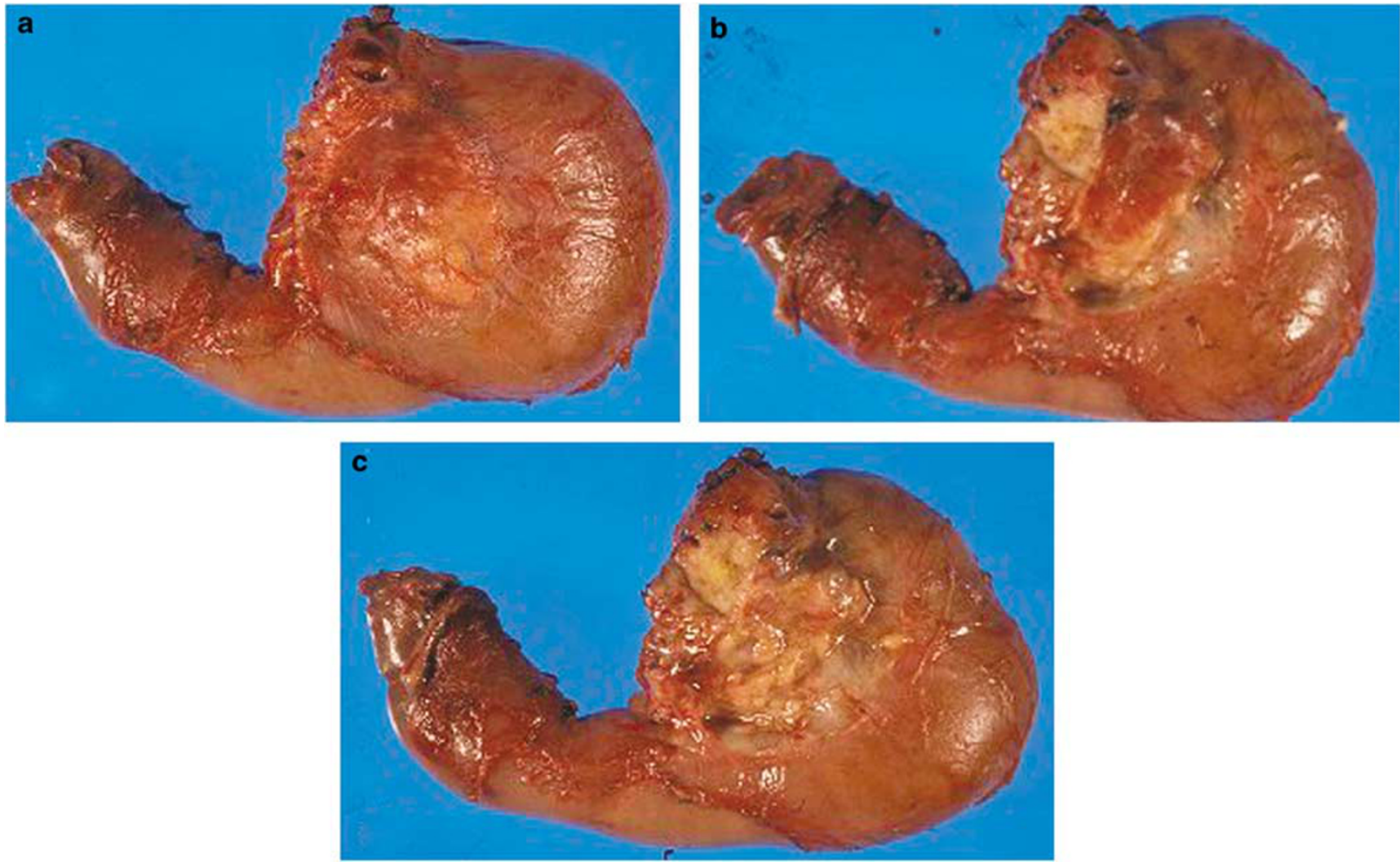

Figure 1 Orange-peeling method. The peripancreatic soft tissues are dissected after margins were obtained but before sectioning of the pancreatic head. (a) Posterior surface of pancreatoduodenectomy specimen is seen before orange peeling. (b) The view after posterior pancreatoduodenal lymph node area (groove between pancreatic head and duodenal wall) is shaved off. (c) The view after posterior pancreatic surface is also orange peeled. 
regions. These arbitrary regions were created (1) to serve as a checklist to ensure removal of all areas, and (2) to determine the yield of lymph nodes in various regions. The eight regions created were pericommon bile duct, anterior pancreatic, anterior pancreatoduodenal, superior pancreatic, inferior pancreatic, posterior pancreatic, posterior pancreatoduodenal, and retroperitoneal margin. The last one was treated separately, inked, cut as a $3-\mathrm{mm}$ thick slice, bread-loafed, and submitted entirely as perpendicular margin (Figure 2). The lymph nodes in the other seven regions were identified, dissected, and submitted in the usual manner. Lymph nodes in the gastric and gall bladder regions or lymph nodes from other sites identified during the surgical procedure and submitted separately for pathologic examination were not included to the count. No special fixative (solutions that highlight and allow the gross identification of even minute lymph nodes) was used, as these are not part of the routine practice.

In both groups, the search for and identification of lymph nodes were mostly performed by the residents, pathology assistants, or student pathology assistants who were on the routine service and have not been informed about the study. They were, however, provided specific instructions regarding the orange-peeling method, and the shaving process was applied in an inclusive manner (Figure 1). Normal tissues were often included in the fragments, and for this reason, the lymph node count was based on the microscopic evaluation, not on macroscopically suspected nodules (potential lymph nodes).

During microscopic evaluation, each face of lymph node was counted as one lymph node as is done routinely, with the exception of the sections from the retroperitoneal margin. This area was evaluated differently because it was submitted entirely for microscopic examination for the purposes of accurate evaluation of this important margin. Although each lymph node face represented an individual lymph node in the other seven regions, the retroperitoneal margin slides had to be assessed differently to avoid counting the same lymph node multiple times. In this region uniform assessment of individual lymph nodes was performed by reconstructing the microscopic slides in consecutive order.

The findings in these cases were compared to those observed in equal numbers of cases $(n=52)$ performed by the same group of surgeons in the respective institutions (Wayne State University and Karmanos Cancer Institute; 26 cases, 1995-1997 and Emory University and Winship Cancer Institute; 26 cases, 2007), as extracted from the surgical pathology reports.

Data were analyzed using the Student's $t$-test and Fisher's exact test to compare the number of lymph nodes and the number of the cases with positive lymph nodes, respectively.

\section{Results}

\section{Number of Lymph Nodes}

The mean number of lymph nodes identified by the orange-peeling approach was 14.1 (median 13, range 5-23, standard deviation 4.8). By contrast, the mean number of lymph nodes identified by the conventional approach was 6.1 (median 5, range 0-20, standard deviation 4.3). The difference was statistically significant $(P=0.0001)$.

For the respective institutions, the means for the orange-peeling approach were 13.8 and 14.4 (medians 15 and 14, ranges 6-20 and 5-23, standard deviations 4.1 and 5.4). By contrast, the means for the conventional approach were 7 and 5.3 , respectively (medians 6.5 and 4, ranges 1-20 and $0-16$, standard deviations 4.1 and 4.4). The differences were statistically significant $(P=0.0001$ and 0.0001$)$.
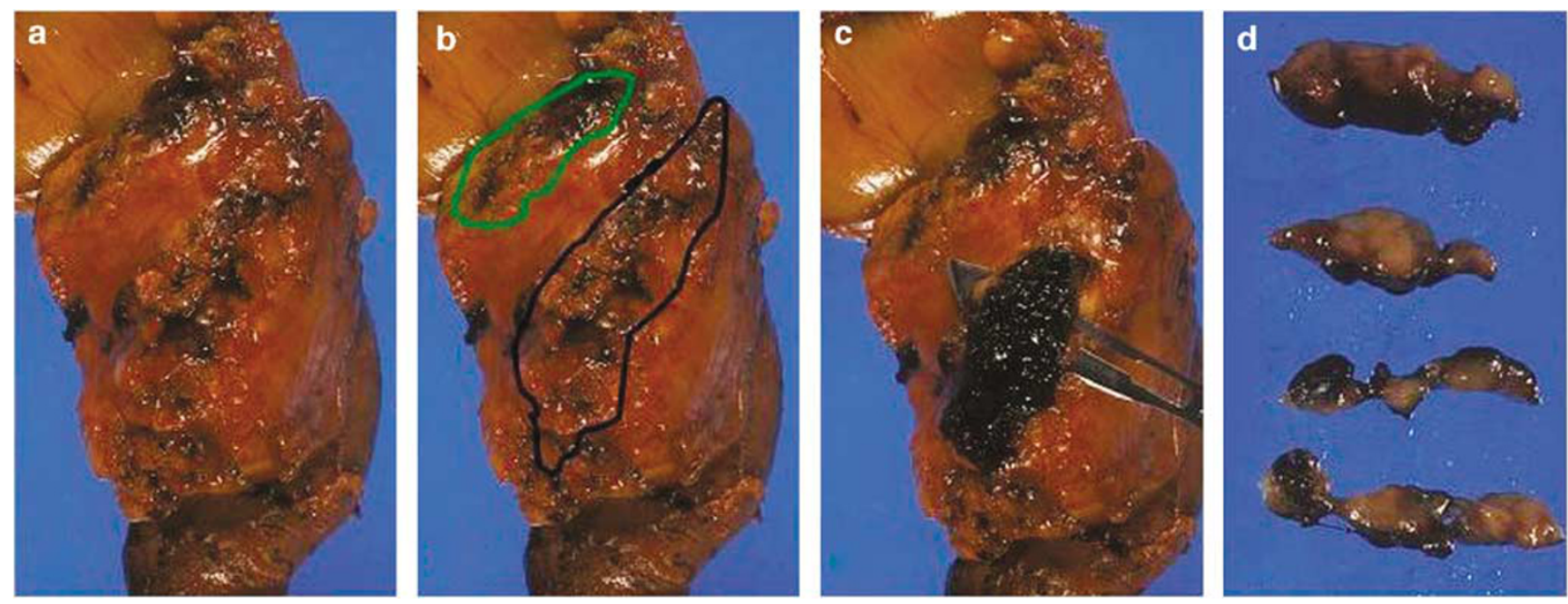

Figure 2 Pancreatic neck margin (green) and retroperitoneal margin (black) are seen before orange peeling (a, b). Retroperitoneal margin is inked black, cut as a 3-mm thick slice (c), bread-loafed, and entirely submitted on edge as perpendicular margin (d). 


\section{Distribution of Lymph Nodes}

Retroperitoneal margin, anterior pancreatoduodenal, and peri-common bile duct regions yielded nodes in $66 \%$ of the cases. An average of three lymph nodes (range 0-15) was identified in the retroperitoneal margin whereas anterior pancreatoduodenal and peri-common bile duct regions each had one lymph node on an average (range $0-6$ and $0-3$, respectively). The posterior pancreatic and anterior pancreatic regions yielded nodes in 63 and 55\% of the cases, respectively. The superior pancreatic region was the most lymph node-poor region, and contained nodes in only $30 \%$ of the cases.

\section{Number of Positive Lymph Nodes}

Total $73 \%$ (38 of 52 cases) of the cases had positive lymph nodes by the orange-peeling approach. By contrast, only $50 \%$ (26 of 52 cases) of the cases had positive lymph nodes by the conventional approach. The difference was statistically significant $(P=0.02)$. For the respective institutions, the percentages for the orange-peeling approach were 88.4 ( 23 of 26 cases) and 58 (15 of 26 cases). By contrast, the percentages for the conventional approach were 54 (14 of 26 cases) and 46 (12 of 26 cases), respectively $(P=0.01$ and 0.5$)$.

The regions most likely to bear positive lymph nodes were the retroperitoneal margin $(26 \%$ of the cases) and posterior pancreatoduodenal region $(26 \%$ of the cases), followed by anterior pancreatic (18\% of the cases), posterior pancreatic (18\% of the cases), superior pancreatic $(15 \%$ of the cases), inferior pancreatic ( $15 \%$ of the cases), anterior pancreatoduodenal (11\% of the cases), and peri-common bile duct regions ( $11 \%$ of the cases), respectively.

Results are summarized in Tables 1 and 2.

\section{Discussion}

This study was performed to assess an improved, feasible method of lymph node harvest in pancreatoduodenectomy specimens of patients with ductal adenocarcinoma of the pancreas. Our results show that an average of 14 lymph nodes is routinely identifiable in conventional pancreatoduodenectomy specimens by incorporating this orange-peeling technique before dissection of the pancreatic head. With this approach, the mean number of lymph nodes found increased from 7 to 13.8 in one institution $(P=0.0001)$ and from 5.3 to 14.4 in another $(P=0.0001)$. In addition, reporting of lymph node-positive cases increased from 54\% (14 of 26 cases) to $88 \%$ (23 of 26 cases) in one $(P=0.02)$ and from $46 \%$ (12 of 26 cases) to $58 \%$ (15 of 26 cases) in the other institution $(P=0.5)$.

We have taken several steps to eliminate potential biases in this study. To this purpose, sampling was performed by residents, pathology assistants, or student pathology assistants during routine practice, with only basic instructions provided. No special fixative was used. It should be noted, however, that there might have been factors positively impacting the number of lymph nodes identified by this method, including the method by which the retroperitoneal (uncinate) margin is sampled. We sample this margin through the approach advocated by Luttges et al. ${ }^{15}$ According to this approach, the retroperitoneal margin is inked, cut as a $3-\mathrm{mm}$ thick slice, bread-loafed, and submitted entirely as a perpendicular margin. This region often contains lymph nodes. In fact, in our study, this was one of the most common regions to contain lymph node as well as to show involvement by tumor (Table 1). As this margin is bread-loafed and submitted entirely, it is occasionally difficult microscopically to distinguish a continuous section of the same lymph node from a separate individual one. This could lead to a slight, false increase in the overall number of identified lymph nodes in some cases. Nevertheless, we believe the impact of this circumstance is minimal, and that there are around 14 lymph nodes in a pancreatoduodenectomy specimen identifiable by this dissection.

In terms of the distribution, in our study, lymph nodes were most abundantly found in the retroperitoneal/uncinate margin, anterior pancreatoduodenal and peri-common bile duct regions. This is in accordance with the previously published data. ${ }^{16}$ The most common sites to harbor a positive lymph node, on the other hand, were the retroperitoneal margin and posterior pancreatoduodenal region. Other studies ${ }^{16,17}$ also documented that in nodepositive ductal adenocarcinoma of the pancreatic head, posterior pancreatoduodenal lymph node seems to be the most common site for nodal involvement.

This study also confirms that if the pancreatoduodenectomy specimens are examined carefully with a method like orange peeling, there is

Table 1 Evaluation of the lymph nodes by orange-peeling method by regions assessed

\begin{tabular}{lccccccc}
\hline & $C B D$ & $A P D$ & $A P$ & $S P$ & IP & $P P$ & $P P D$ \\
\hline Cases with lymph nodes (\%) & 66 & 66 & 55 & 30 & 42 & 63 & 51 \\
Cases with positive lymph nodes (\%) & 11 & 11 & 18 & 15 & 15 & 18 & 26 \\
\hline
\end{tabular}

AP, anterior pancreatic; APD, anterior pancreatoduodenal; CBD, common bile duct; IP, inferior pancreatic; PP, posterior pancreatic; PPD, posterior pancreatoduodenal; RM, retroperitoneal margin; SP, superior pancreatic. 
Table 2 Comparison of orange-peeling method with nonspecific method by institution

\begin{tabular}{|c|c|c|c|c|c|c|}
\hline & \multicolumn{3}{|c|}{ Orange-peeling method } & \multicolumn{3}{|c|}{ Conventional method } \\
\hline & Overall & $W S U^{\mathrm{a}}$ & Emory $^{\mathrm{b}}$ & Overall & $W S U^{\mathrm{a}}$ & Emory $^{\mathrm{b}}$ \\
\hline Median no. of lymph nodes identified & 13 & 15 & 14 & 5 & 6.5 & 4 \\
\hline Mean no. of lymph nodes identified & 14.1 & 13.8 & 14.4 & 6.1 & 7 & 5.3 \\
\hline Range of no. of lymph nodes & $5-23$ & $6-20$ & $5-23$ & $0-20$ & $1-20$ & $0-16$ \\
\hline$\%$ of cases with $(+)$ lymph nodes & $73(38 / 52)$ & $88(23 / 26)$ & $58(15 / 26)$ & $50(26 / 52)$ & $54(14 / 26)$ & $46(12 / 26)$ \\
\hline
\end{tabular}

${ }^{\mathrm{a}}$ Wayne State University School of Medicine, Karmanos Cancer Institute.

${ }^{\mathrm{b}}$ Emory University School of Medicine, Winship Cancer Institute.

metastasis to the lymph nodes in most patients $(72 \%)$. This is important not only clinically (for staging, therapeutic and prognostic purposes) but also for researchers, because it supports the hypothesis that one of the reasons for the dismal outcome of ductal adenocarcinoma is the delay in diagnosis, an issue that may have been underevidenced in some other studies due to inadequate sampling of lymph nodes. Some authors reported that the greater the number of lymph nodes resected, the greater the possibility of detecting positive nodes in gastrointestinal (GI) cancers. ${ }^{18,19}$ Therefore, tumor staging can be significantly affected by the extent of lymphadenectomy. Moreover, there is a growing body of evidence demonstrating that the number of involved nodes or the metastatic/resected lymph node ratio could be a significant prognostic factor in human malignancies. ${ }^{14,19-24}$ In fact, because of these observations, there is a trend to treat the GI cancer cases with inadequate number of lymph nodes as if they are lymph node positive even if these few lymph nodes do not show any evidence of metastasis.

Currently there is no consensus on the optimal number of lymph nodes to examine to stage accurately node-negative ductal adenocarcinoma after pancreatoduodenectomy. In a publication by the Royal College of Pathologists outlining standards in the histopathologic analysis of exocrine tumors of the pancreas, it is stated that 10-20 nodes should be available for examination within a pancreatoduodenectomy specimen ${ }^{25}$ but no literature is provided to substantiate this observation. In their study of the SEER database, Tomlinson et $a l^{12}$ demonstrated that dichotomizing the pNo cohort on 15 or more examined lymph nodes resulted in the most statistically significant survival difference. However, it is important to note that the median number of lymph nodes identified in this database was 7. Similarly, in their analysis of SEER data, Slidell et $a l^{8}$ disclosed that the percentage of cases reported to have 15 or more nodes was only $19 \%$. This casts doubts on whether the examination of 15 lymph nodes is achievable in the majority of pancreatoduodenectomy specimens. ${ }^{8}$

Recent reports from major US medical centers including Johns Hopkins ${ }^{13}$ and Memorial SloanKettering Cancer Center $^{14}$ suggest that the median number of lymph nodes can be as high as 17; although these studies appear to have documented the overall lymph node count, including all lymph nodes harvested from their patients presumably including, gastric, celiac, and gallbladder regions, and some of their patients were total pancreatectomies. However, in our analysis focusing only on the pancreatoduodenectomy specimens (lymph nodes in the gastric and gall bladder regions or lymph nodes from other sites identified during the surgical procedure and submitted separately for pathologic examination are not included to the count), by incorporating this orange-peeling technique, an average of 14 lymph nodes were routinely identified. More than 12 lymph nodes were identified in $83 \%$ of the cases.

It is becoming increasingly clear that identifying adequate lymph nodes is essential for accurate staging of pancreas cancer and patient stratification. The study from Memorial Sloan-Kettering Cancer Center also showed that patients who were deemed node negative with fewer than 12 lymph node assessed had a similar survival to patients with a single positive lymph node and more than 12 lymph nodes assessed. With more extensive nodal assessment (ie, $\geq 12$ lymph node assessed), patients with node-negative disease had improved survival compared to those with a single positive lymph node. ${ }^{14}$ This observation may provide an explanation for previous reports that have failed to demonstrate a significant survival difference between patients with a single lymph node metastasis and those with No disease. ${ }^{26,27}$ Another interesting finding from the MSKCC data was that the survival of No patients who had fewer than 12 lymph nodes evaluated at the time of pancreatoduodenectomy tended to be shorter than the survival of No patients who had at least 12 lymph nodes examined. ${ }^{14}$ Berger et al ${ }^{28}$ also reported that No patients with fewer than 12 lymph nodes had a worse prognosis.

In summary, inadequate surgical lymphadenectomy and/or pathologic assessment appears to understage No patients as they have similar outcomes to patients with a single positive lymph node out of a minimum of 12 lymph nodes assessed. ${ }^{14,28}$ Our results indicate that on average, 14 lymph nodes (median 13) are identifiable by orange-peeling method. However, this number is attainable only by a careful dissection of peripancreatic soft tissue 
and separation of potential lymph node regions before sectioning the pancreatic head, which is the conventional approach applied in most institutions, through which only a mean of six lymph nodes has been documented in the authors' institutions. Therefore, although the orange-peeling approach increased the number of slides submitted for examination of the lymph node, this is not a substantial burden compared to the importance of the information obtained and it is strongly advisable that in the gross laboratories, lymph node identification is performed before the sectioning of pancreatic head to accurately identify all the lymph nodes.

\section{Acknowledgement}

This study was supported in part by National Cancer Institute Specialized Program in Research Excellence (SPORE), P50-CA62924.

\section{References}

1 Jemal A, Siegel R, Ward E, et al. Cancer statistics, 2006. CA Cancer J Clin 2006;56:106-130.

2 Lim JE, Chien MW, Earle CC. Prognostic factors following curative resection for pancreatic adenocarcinoma: a population-based, linked database analysis of 396 patients. Ann Surg 2003;237:74-85.

3 Pawlik TM, Abdalla EK, Barnett CC, et al. Feasibility of a randomized trial of extended lymphadenectomy for pancreatic cancer. Arch Surg 2005;140:584-589.

4 Yeo CJ, Cameron JL, Sohn TA, et al. Six hundred fifty consecutive pancreaticoduodenectomies in the 1990s: pathology, complications, and outcomes. Ann Surg 1997;226:248-257.

5 Benassai G, Mastrorilli M, Mosella F, et al. Significance of lymph node metastases in the surgical management of pancreatic head carcinoma. J Exp Clin Cancer Res 1999;18:23-28.

6 Breslin TM, Hess KR, Harbison DB, et al. Neoadjuvant chemoradiotherapy for adenocarcinoma of the pancreas: treatment variables and survival duration. Ann Surg Oncol 2001;8:123-132.

7 Gebhardt C, Meyer W, Reichel M, et al. Prognostic factors in the operative treatment of ductal pancreatic carcinoma. Langenbecks Arch Surg 2000;385:14-20.

8 Slidell MB, Chang DC, Cameron JL, et al. Impact of total lymph node count and lymph node ratio on staging and survival after pancreatectomy for pancreatic adenocarcinoma: a large, population-based analysis. Ann Surg Oncol 2007;15:165-174.

9 Sohn TA, Yeo CJ, Cameron JL, et al. Resected adenocarcinoma of the pancreas-616 patients: results, outcomes, and prognostic indicators. J Gastrointest Surg 2000;4:567-579.

10 Reber HA. Lymph node involvement as a prognostic factor in pancreatic cancer. Int $\mathrm{J}$ Pancreatol 1990;7:125-127.

11 Staley CA, Cleary KR, Abbruzzese JL, et al. The need for standardized pathologic staging of pancreaticoduodenectomy specimens. Pancreas 1996;12:373-380.

12 Tomlinson JS, Jain S, Bentrem DJ, et al. Accuracy of staging node-negative pancreas cancer: a potential quality measure. Arch Surg 2007;142:767-773.
13 Pawlik TM, Gleisner AL, Cameron JL, et al. Prognostic relevance of lymph node ratio following pancreaticoduodenectomy for pancreatic cancer. Surgery 2007;141:610-618.

14 House MG, Gonen M, Jarnagin WR, et al. Prognostic significance of pathologic nodal status in patients with resected pancreatic cancer. J Gastrointest Surg 2007;11:1549-1555.

15 Luttges J, Vogel I, Menke M, et al. The retroperitoneal resection margin and vessel involvement are important factors determining survival after pancreaticoduodenectomy for ductal adenocarcinoma of the head of the pancreas. Virchows Arch 1998;433:237-242.

16 Nagakawa T, Kobayashi H, Ueno K, et al. The pattern of lymph node involvement in carcinoma of the head of the pancreas. A histologic study of the surgical findings in patients undergoing extensive nodal dissections. Int J Pancreatol 1993;13:15-22.

17 Schwarz RE, Smith DD. Extent of lymph node retrieval and pancreatic cancer survival: information from a large US population database. Ann Surg Oncol 2006;13:1189-1200.

18 Braat AE, Oosterhuis JW, de Vries JE, et al. Lymphatic staging in colorectal cancer: pathologic, molecular, and sentinel node techniques. Dis Colon Rectum 2005;48:371-383.

19 Compton CC, Greene FL. The staging of colorectal cancer: 2004 and beyond. CA Cancer J Clin 2004;54:295-308.

20 Hayashi H, Ochiai T, Suzuki T, et al. Superiority of a new UICC-TNM staging system for gastric carcinoma. Surgery 2000;127:129-135.

21 Kunisaki C, Shimada H, Nomura M, et al. Comparative evaluation of gastric carcinoma staging: Japanese classification versus New American Joint Committee on Cancer/International Union Against Cancer Classification. Ann Surg Oncol 2004;11:203-206.

22 van der Wal BC, Butzelaar RM, van der Meij S, et al. Axillary lymph node ratio and total number of removed lymph nodes: predictors of survival in stage I and II breast cancer. Eur J Surg Oncol 2002;28: 481-489.

23 Megale Costa LJ, Soares HP, Gaspar HA, et al. Ratio between positive lymph nodes and total dissected axillaries lymph nodes as an independent prognostic factor for disease-free survival in patients with breast cancer. Am J Clin Oncol 2004;27:304-306.

24 Nitti D, Marchet A, Olivieri M, et al. Ratio between metastatic and examined lymph nodes is an independent prognostic factor after D2 resection for gastric cancer: analysis of a large European monoinstitutional experience. Ann Surg Oncol 2003;10:1077-1085.

25 Royal College of Pathologists. Royal College of Pathologists Minimum Dataset for the Histopathological Reporting of Pancreatic, Ampulla of Vater and Bile Duct Carcinoma. Royal College of Pathologists: London, 2002.

26 Ishikawa O, Ohigashi $\mathrm{H}$, Sasaki Y, et al. Practical grouping of positive lymph nodes in pancreatic head cancer treated by an extended pancreatectomy. Surgery 1997;121:244-249.

27 Hermanek P. Staging of exocrine pancreatic carcinoma. Eur J Surg Oncol 1991;17:167-172.

28 Berger AC, Watson JC, Ross EA, et al. The metastatic/ examined lymph node ratio is an important prognostic factor after pancreaticoduodenectomy for pancreatic adenocarcinoma. Am Surg 2004;70:235-240. 